\title{
Evaluation of milk thistle (Silybum marianum (L.) Gaertn.) seed germination in relation to seed health and seedling emergence
}

\author{
AGNIESZKA ROSIŃSKA ${ }^{* 1}$, HANNA DORNA ${ }^{1}$, DOROTA SZOPIŃSKA ${ }^{1}$, LIDIA IRZYKOWSKA ${ }^{1}$, \\ KATARZYNA SEIDLER-ŁOŻYKOWSKA ${ }^{2}$
}

${ }^{1}$ Department of Phytopathology, Seed Science and Technology

Faculty of Horticulture and Landscape Architecture

Poznań University of Life Sciences

Dąbrowskiego 159

60-594 Poznań, Poland

${ }^{2}$ Institute of Natural Fibres and Medicinal Plants

Kolejowa 2

62-630 Plewiska, Poland

* corresponding author: tel.: +48 618487 926, e-mail: agnieszka.rosinska@up.poznan.pl

\section{Summary}

Introduction: : Milk thistle (Silybum marianum (L.) Gaertn.) is an important medicinal plant. Achenes of milk thistle contain sylimarin, protecting liver cells against toxic compounds.

Objective: The aim of the research was to find an optimum method of evaluation of milk thistle seed germination.

Methods: Ten seed samples were tested. The seeds were germinated: on top of blotter paper, on top of blotter paper after seed disinfection, between pleated blotter paper, in rolled blotter paper and in sand. Germination at the first and final counts, the percentages of abnormal seedlings and dead seeds were determined. The correlation coefficients between seed germination, evaluated with various methods, and seedling emergence were calculated. Moreover, fungi associated with seeds and diseased seedlings were identified.

Results: The lowest percentage of normal seedlings was observed after germination on the top of blotter. Highly significant positive correlations were noted between seedling emergence and seed germination at the final count evaluated in rolled paper, between pleated paper and in sand. The fungi from genera: 
Alternaria, Fusarium, Penicillium, Trichoderma, Ulocladium and Verticillium were frequently identified on seeds and seedlings.

Conclusions: Infestation with fungi significantly affected milk thistle seed germination and plant emergence. Germination in rolled blotter paper may be recommended for evaluation of milk thistle seed germination, as the most practical and significantly correlated with seedling emergence.

Key words: milk thistle, seed germination, seedling emergence, seedling health

Słowa kluczowe: ostropest plamisty, kiełkowanie nasion, wschody roślin, zdrowotność siewek

\section{INTRODUCTION}

Milk thistle (Silybum marianum (L.) Gaertn.) is an important medicinal plant belonging to Asteraceae family, commercially cultivated for fruits - achenes, termed in practice as seeds. They contain a complex of flavonolignans (sylimarin) used in medicine for treatment of liver diseases, such as jaundice, bile stones, hepatitis and steatosis [1]. Dietary supplements containing the extract of milk thistle fruits are the best-selling plant pharmaceuticals in the United States and Europe. Poland is one of the most important producers of raw material and medicinal preparations based on the extract from milk thistle fruits in Europe. Production of this plant is located mainly in Wielkopolska, Kujawy, Żuławy and Lublin regions, on an area of approximately $1500 \mathrm{ha}$. The annual market requirement for milk thistle seeds in Poland is $30000 \mathrm{~kg}[2,3]$.

The germination test is performed to determine the germination potential of a seed lot. It allows to compare the quality of different seed samples and estimate their field planting value [4]. International Seed Testing Association (ISTA) recommends to germinate milk thistle seeds on top of paper or between paper at constant or alternating temperatures, $20^{\circ} \mathrm{C}$ or $20 / 30^{\circ} \mathrm{C}$, respectively. Moreover, germination in sand is advised in case of low germination at the final count, caused by spreading of seed-borne fungi between seeds or seedlings on a paper medium. Seeds of this plant can be infested with numerous fungi, such as: Alternaria alternata (Fr.) Keissler, Aspergillus flavus Link., A. fumigatus Fresen, A. niger Tiegh., Bipolaris sp., Botrytis cinerea Pers, Drechslera sp., Cladosporium cladosporioides (Fresen.) G.A. de Vries, Epicoccum nigrum Link, Fusarium sporotrichioides Sherb., Fusarium spp., Gibberella avenacea R.J. Cook (formely Fusarium avenaceum (Fr.) Sacc.), Mucor hiemalis Wehmer, Penicillium chrysogenum Thom, P. decumbens Thom, Penicillium spp., Rhizopus stolonifer (Ehrenb.) Vuill. (formely Rhizopus nigricans
Ehrenberg), Sarocladium strictum (W. Gams) Summerb, (formely Cephalosporium acremonium Corda), Stemphylium botryosum Wallr., Trichothecium roseum (Pers.) Link, Ulocladium spp. and Verticillium spp. $[5,6]$. Some of these fungi, for instance A. alternata, may occur on all tested seeds from the sample [personal observation]. High humidity and limited space, along with germination on top of blotter in Petri dishes or on the Jacobsen table, favor the growth and spread of fungi. It frequently results in numerous secondary seedling infections. Hence, the evaluation of these seedlings is difficult and results of the tests are often not reliable. Nevertheless, germination on top of paper is routinely used by many seed testing laboratories. Therefore, the aim of the experiment was to indicate the optimum method of milk thistle seed germination testing, considering the influence of seed-associated fungi on seed germination and seedling emergence.

\section{MATERIAL AND METHODS}

In the experiment, ten seed samples of milk thistle (Silybum marianum (L.) Gaertn.) cv. Silma (I$\mathrm{X}$ ), obtained from Poznańskie Zakłady Zielarskie "Herbapol" SA, were tested. The seeds were multiplied in the area of Wielkopolskie voivodship in 2011 and examined directly after receiving from the company i.e. approximately six months after the harvest.

\section{Seed germination tests}

In germination tests blotter (paper weight $65 \mathrm{~g} / \mathrm{m}^{2}$ ) and medium flow rate was used. Seed germination was evaluated: on top of paper, on top of paper after seed disinfection, between pleated paper, in rolled paper and in sand. For each method, 400 seeds (four replicates of 100 seeds) from each sample were 
tested. Regardless of the applied method, seeds were incubated for 21 days at $20^{\circ} \mathrm{C}$ in darkness. Germination at the first count was determined after 7 days, whereas after 21 days of germination at the final count, the percentages of abnormal diseased and deformed seedlings as well as fresh and dead seeds were evaluated.

Germination on top of paper (TP). The seeds were placed in $9 \mathrm{~cm}$ diameter Petri dishes $(20$ seeds per dish), on six layers of blotter paper moistened with distilled water.

Germination on top of paper after seed disinfection (TP-D). The seeds were disinfected with $1 \%$ aqueous solution of sodium hypochlorite $(\mathrm{NaClO})$ for $10 \mathrm{~min}$, next rinsed three times with sterile distilled water and surface dried on sterile blotter paper. After disinfection seeds were placed in $9 \mathrm{~cm}$ diameter Petri dishes (20 seeds per dish), on six layers of blotter paper moistened with distilled water.

Germination between pleated paper (PP). The seeds were placed in a pleated, accordion-like blotter paper stripes with 50 pleats (one seed per pleat). Each pleated strip was made of two layers of blotter paper, $12 \times 200 \mathrm{~cm}$ in size, placed in plastic box $(12.5 \times 21 \mathrm{~cm})$ and moistened with distilled water. The boxes during incubation were tightly closed.

Germination in rolled paper (RP). The seeds were placed in rolled blotter paper (50 seeds per roll). Each roll was made of three layers of blotter paper, $450 \times 280 \mathrm{~cm}$ in size. First two layers were moistened with distilled water, then the seeds were spaced uniformly and covered with another layer of blotter paper. Subsequently, blotter sheets were rolled and wrapped in plastic bags. During incubation the rolls were kept in closed containers in an upright position.

Germination in sand (S). Sterilized river sand was placed in plastic containers, $15 \times 21 \mathrm{~cm}$ in size, and then moistened with distilled water to $65 \%$ of its water holding capacity. The seeds (50 seeds per container) were planted on the $4 \mathrm{~cm}$ layer of sand and covered with another $1.5 \mathrm{~cm}$ layer. The containers were not covered, therefore, to prevent the sand from drying out, it was sprayed daily with distilled water.

\section{Seed health test}

Deep-freeze blotter test was performed to evaluate seed infestation with fungi. From each sample, 200 seeds were tested (four replicates of 50 seeds). The seeds were placed on blotter moistened with distilled water in $9 \mathrm{~cm}$ diameter Petri dishes (10 seeds per plate). Primarily, seeds were incubated for 2 days in darkness at $20^{\circ} \mathrm{C}$, next frozen for $24 \mathrm{~h}$ at $-20^{\circ} \mathrm{C}$, and successively incubated for 7 days at $20^{\circ} \mathrm{C}$, under $12 \mathrm{~h}$ alternating cycle of darkness and NUV light. Infestation of seeds with fungi was evaluated on the basis of their growth and sporulation, using stereomicroscope (50-60x magnification) and compound microscope (400x magnification) when needed [7-9].

\section{Seedling emergence test}

Seedling emergence test was carried out in AURA commercial soil, produced by Hollas Company. In the experiment, 200 seeds (four replicates of 50 seeds) from each sample were sown in seeding trays filled with growing media moistened with distilled water. Seeding trays were incubated in a growth chamber in $12 / 12 \mathrm{~h}$ light period ( $12 \mathrm{~h}$ of daylight and $12 \mathrm{~h}$ of darkness) at $20^{\circ} \mathrm{C}$. The seedlings were sprinkled with distilled water as needed. Emerged seedlings were counted daily for 21 days. Infected seedlings were successively removed from trays, rinsed under tap water and with sterile distilled water, and next incubated in $9 \mathrm{~cm}$ diameter Petri dishes on six layers of blotter paper moistened with distilled water (two seedlings per plate) for 10 days, under $12 \mathrm{~h}$ alternating cycles of NUV light and darkness. After incubation the fungi growing on seedlings were identified on the basis of their growth and sporulation using a stereomicroscope (magnification 50-60x) [7-9].

\section{Statistical analysis}

The obtained results were evaluated by analysis of followed by the Duncan's multiple range test at $\alpha=0.05$ [10] using STAT software. Parameters describing seed vigour were calculated using SeedCalculator 2.1 [11]. To determine the relationship between germination at the final count, evaluated by individual tests, and seedling emergence, linear regression analysis was performed and correlation coefficients ( $r$ ) were calculated.

Ethical approval: The conducted research is not related to either human or animal use.

\section{RESULTS AND DISCUSSION}

In general, the lowest values of seed germination at the first and final counts, connected with the 
Table 1

Comparison of methods of milk thistle seed germination

\begin{tabular}{|c|c|c|c|c|c|c|}
\hline \multirow[b]{2}{*}{ Method } & \multirow[b]{2}{*}{$\begin{array}{c}\text { Germination at the first count } \\
\text { [\%] }\end{array}$} & \multirow[b]{2}{*}{$\begin{array}{c}\text { Germination at the final count } \\
{[\%]}\end{array}$} & \multicolumn{2}{|c|}{ Abnormal seedlings } & \multirow[b]{2}{*}{$\begin{array}{c}\text { Fresh seeds } \\
{[\%]}\end{array}$} & \multirow[b]{2}{*}{$\begin{array}{c}\text { Dead seeds } \\
{[\%]}\end{array}$} \\
\hline & & & $\begin{array}{c}\text { Infected } \\
{[\%]}\end{array}$ & $\begin{array}{l}\text { Deformated } \\
{[\%]}\end{array}$ & & \\
\hline 1 & 2 & 3 & 4 & 5 & 6 & 7 \\
\hline \multicolumn{7}{|c|}{ Sample I } \\
\hline $\mathrm{TP}^{*}$ & $15.8 \mathrm{a}$ & $22.3 \mathrm{a}$ & $43.3 \mathrm{~d}$ & $0.3 \mathrm{a}$ & $0 \mathrm{a}$ & $34.3 \mathrm{~b}$ \\
\hline TP-D & $18.3 \mathrm{a}$ & $21.5 \mathrm{a}$ & $40.0 \mathrm{~d}$ & $0.8 \mathrm{ab}$ & $0 \mathrm{a}$ & $37.8 \mathrm{~b}$ \\
\hline PP & $39.0 \mathrm{~b}$ & $40.8 \mathrm{~b}$ & $12.3 \mathrm{~b}$ & $2.3 \mathrm{c}$ & $0.8 \mathrm{a}$ & $44.0 \mathrm{c}$ \\
\hline $\mathrm{RP}$ & $39.3 \mathrm{~b}$ & $40.5 \mathrm{~b}$ & $21.8 \mathrm{c}$ & $2.0 \mathrm{bc}$ & $0.5 \mathrm{a}$ & $35.3 \mathrm{~b}$ \\
\hline S & $49.8 \mathrm{c}$ & $56.8 \mathrm{c}$ & $5.8 \mathrm{a}$ & $0.3 \mathrm{a}$ & $24.5 \mathrm{~b}$ & $12.3 \mathrm{a}$ \\
\hline \multicolumn{7}{|c|}{ Sample II } \\
\hline TP & $15.5 \mathrm{a}$ & $18.8 \mathrm{a}$ & $53.0 \mathrm{c}$ & $0 \mathrm{a}$ & $0 \mathrm{a}$ & $28.3 \mathrm{a}$ \\
\hline TP-D & $16.8 \mathrm{a}$ & $21.0 \mathrm{a}$ & $52.0 \mathrm{c}$ & $0 \mathrm{a}$ & $0 \mathrm{a}$ & $27.0 \mathrm{a}$ \\
\hline PP & $55.0 \mathrm{~d}$ & $55.8 \mathrm{c}$ & $24.3 \mathrm{~b}$ & $1.8 \mathrm{~b}$ & $0 \mathrm{a}$ & $18.3 \mathrm{a}$ \\
\hline $\mathrm{RP}$ & $47.0 \mathrm{c}$ & $47.8 \mathrm{~b}$ & $28.8 \mathrm{~b}$ & $2.8 \mathrm{~b}$ & $0.3 \mathrm{a}$ & $20.5 \mathrm{a}$ \\
\hline$S$ & $32.0 \mathrm{~b}$ & $53.3 \mathrm{bc}$ & $7.0 \mathrm{a}$ & $1.5 \mathrm{~b}$ & $19.5 \mathrm{~b}$ & $18.8 \mathrm{a}$ \\
\hline \multicolumn{7}{|c|}{ Sample III } \\
\hline TP & $25.5 \mathrm{~b}$ & $29.8 \mathrm{a}$ & $58.3 \mathrm{~b}$ & $0.3 \mathrm{a}$ & $0 \mathrm{a}$ & $11.8 \mathrm{a}$ \\
\hline TP-D & $17.3 \mathrm{a}$ & $24.5 \mathrm{a}$ & $61.5 \mathrm{~b}$ & $0.5 \mathrm{a}$ & $0 \mathrm{a}$ & $13.5 \mathrm{a}$ \\
\hline $\mathrm{PP}$ & $54.3 \mathrm{~d}$ & $54.8 \mathrm{~b}$ & $20.5 \mathrm{a}$ & $2.5 \mathrm{~b}$ & $2.3 \mathrm{~b}$ & $20.0 \mathrm{~b}$ \\
\hline $\mathrm{RP}$ & $66.0 \mathrm{e}$ & $67.0 \mathrm{c}$ & $12.8 \mathrm{a}$ & $4.0 \mathrm{~b}$ & $1.0 \mathrm{ab}$ & $15.3 \mathrm{ab}$ \\
\hline S & $54.3 \mathrm{c}$ & $62.8 \mathrm{bc}$ & $14.5 \mathrm{a}$ & $1.5 \mathrm{ab}$ & $12.3 \mathrm{c}$ & $9.0 \mathrm{a}$ \\
\hline \multicolumn{7}{|c|}{ Sample IV } \\
\hline TP & $29.0 \mathrm{a}$ & $42.5 \mathrm{ab}$ & $33.8 \mathrm{c}$ & $0.5 \mathrm{a}$ & $0 \mathrm{a}$ & $23.3 \mathrm{a}$ \\
\hline TP-D & $30.0 \mathrm{a}$ & $40.5 \mathrm{a}$ & $40.0 \mathrm{c}$ & $0 \mathrm{a}$ & $0 \mathrm{a}$ & $19.5 \mathrm{a}$ \\
\hline $\mathrm{PP}$ & $56.8 \mathrm{~b}$ & $59.0 \mathrm{c}$ & $20.8 \mathrm{~b}$ & $2.8 \mathrm{~b}$ & $0.5 \mathrm{a}$ & $17.0 \mathrm{a}$ \\
\hline $\mathrm{RP}$ & $60.3 \mathrm{~b}$ & $61.8 \mathrm{c}$ & $9.8 \mathrm{a}$ & $5.0 \mathrm{~b}$ & $0.3 \mathrm{a}$ & $23.3 \mathrm{a}$ \\
\hline S & $49.3 \mathrm{~b}$ & $55.5 \mathrm{bc}$ & $16.3 \mathrm{ab}$ & $2.3 \mathrm{~b}$ & $5.0 \mathrm{~b}$ & $21.0 \mathrm{a}$ \\
\hline \multicolumn{7}{|c|}{ Sample V } \\
\hline TP & $28.0 \mathrm{a}$ & $40.5 \mathrm{a}$ & $41.3 \mathrm{c}$ & $0 \mathrm{a}$ & $0 \mathrm{a}$ & $18.3 \mathrm{c}$ \\
\hline TP-D & $31.5 \mathrm{a}$ & $41.8 \mathrm{a}$ & $48.3 c$ & $0 \mathrm{a}$ & $0 \mathrm{a}$ & $10.0 \mathrm{ab}$ \\
\hline $\mathrm{PP}$ & $58.8 \mathrm{~b}$ & $59.5 \mathrm{~b}$ & $26.3 \mathrm{~b}$ & $2.5 \mathrm{bc}$ & $0.5 \mathrm{a}$ & $11.3 \mathrm{~b}$ \\
\hline $\mathrm{RP}$ & $79.3 \mathrm{c}$ & $79.3 c$ & $11.5 \mathrm{a}$ & $3.8 \mathrm{c}$ & $0.8 \mathrm{a}$ & $4.8 \mathrm{a}$ \\
\hline$S$ & $56.3 \mathrm{~b}$ & $59.8 \mathrm{~b}$ & $12.8 \mathrm{a}$ & $1.3 \mathrm{~b}$ & $9.8 \mathrm{~b}$ & $16.5 \mathrm{ab}$ \\
\hline \multicolumn{7}{|c|}{ Sample VI } \\
\hline $\mathrm{TP}$ & $7.5 \mathrm{a}$ & $16.5 \mathrm{a}$ & $52.8 \mathrm{c}$ & $0 \mathrm{a}$ & $0 \mathrm{a}$ & $30.8 \mathrm{a}$ \\
\hline TP-D & $12.8 \mathrm{a}$ & $22.0 \mathrm{a}$ & $43.5 \mathrm{c}$ & $0.8 \mathrm{a}$ & $0.5 \mathrm{a}$ & $33.3 \mathrm{a}$ \\
\hline $\mathrm{PP}$ & $47.8 \mathrm{c}$ & $50.0 \mathrm{c}$ & $17.3 \mathrm{ab}$ & $6.0 \mathrm{~b}$ & $3.3 \mathrm{~b}$ & $23.5 \mathrm{a}$ \\
\hline $\mathrm{RP}$ & $53.3 \mathrm{c}$ & $55.3 \mathrm{c}$ & $12.8 \mathrm{a}$ & $5.0 \mathrm{~b}$ & $3.5 \mathrm{~b}$ & $23.5 \mathrm{a}$ \\
\hline$S$ & $26.8 \mathrm{~b}$ & $34.5 \mathrm{~b}$ & $22.0 \mathrm{~b}$ & $0.5 \mathrm{a}$ & $16.5 \mathrm{c}$ & $26.5 \mathrm{a}$ \\
\hline \multicolumn{7}{|c|}{ Sample VII } \\
\hline $\mathrm{TP}$ & $2.8 \mathrm{a}$ & $7.8 \mathrm{~b}$ & $11.5 \mathrm{~b}$ & $0.8 \mathrm{a}$ & $0 \mathrm{a}$ & $80.0 \mathrm{bc}$ \\
\hline TP-D & $1.8 \mathrm{a}$ & $9.0 \mathrm{~b}$ & $5.0 \mathrm{a}$ & $0.8 \mathrm{a}$ & $2.3 \mathrm{~b}$ & $83.0 \mathrm{c}$ \\
\hline $\mathrm{PP}$ & $8.0 \mathrm{~b}$ & $9.0 \mathrm{~b}$ & $4.3 \mathrm{a}$ & $3.3 \mathrm{~b}$ & $13.8 \mathrm{~d}$ & $69.8 \mathrm{~b}$ \\
\hline $\mathrm{RP}$ & $8.5 \mathrm{~b}$ & $9.5 \mathrm{~b}$ & $2.3 \mathrm{a}$ & $2.3 \mathrm{~b}$ & $6.3 c$ & $79.8 \mathrm{bc}$ \\
\hline$S$ & $1.3 \mathrm{a}$ & $2.8 \mathrm{a}$ & $9.0 \mathrm{~b}$ & $0.0 \mathrm{a}$ & $53.0 \mathrm{e}$ & $35.3 \mathrm{a}$ \\
\hline \multicolumn{7}{|c|}{ Sample VIII } \\
\hline $\mathrm{TP}$ & $11.8 \mathrm{a}$ & $14.3 \mathrm{a}$ & $66.8 \mathrm{c}$ & $0.3 \mathrm{a}$ & $0 \mathrm{a}$ & $18.8 \mathrm{~b}$ \\
\hline TP-D & $11.0 \mathrm{a}$ & $15.3 \mathrm{a}$ & $63.5 c$ & $0 \mathrm{a}$ & $0 \mathrm{a}$ & $21.3 \mathrm{~b}$ \\
\hline PP & $49.5 \mathrm{~b}$ & $50.0 \mathrm{~b}$ & $30.8 \mathrm{~b}$ & $1.8 \mathrm{~b}$ & $0.3 \mathrm{a}$ & $17.3 \mathrm{~b}$ \\
\hline $\mathrm{RP}$ & $56.8 \mathrm{~b}$ & $57.5 \mathrm{c}$ & $20.0 \mathrm{a}$ & $2.0 \mathrm{~b}$ & $0.3 \mathrm{a}$ & $20.3 \mathrm{~b}$ \\
\hline S & $52.5 \mathrm{~b}$ & $56.3 \mathrm{bc}$ & $24.3 \mathrm{ab}$ & $0.5 \mathrm{a}$ & $9.0 \mathrm{~b}$ & $10.0 \mathrm{a}$ \\
\hline
\end{tabular}




\begin{tabular}{|c|c|c|c|c|c|c|}
\hline \multicolumn{7}{|c|}{ Sample IX } \\
\hline $\mathrm{TP}$ & $17.0 \mathrm{ab}$ & $25.8 \mathrm{ab}$ & $49.0 \mathrm{c}$ & $1.5 \mathrm{~b}$ & $0 \mathrm{a}$ & $23.8 \mathrm{a}$ \\
\hline TP-D & $10.8 \mathrm{a}$ & $21.5 \mathrm{a}$ & $55.3 \mathrm{c}$ & $1.0 \mathrm{~b}$ & $0 \mathrm{a}$ & $22.3 \mathrm{a}$ \\
\hline $\mathrm{PP}$ & $56.0 \mathrm{~d}$ & $58.5 \mathrm{~d}$ & $15.5 \mathrm{a}$ & $4.8 \mathrm{c}$ & $2.0 \mathrm{c}$ & $19.3 \mathrm{a}$ \\
\hline $\mathrm{RP}$ & $44.5 \mathrm{c}$ & $46.5 \mathrm{c}$ & $27.3 \mathrm{~b}$ & $5.8 \mathrm{c}$ & $0.5 \mathrm{~b}$ & $20.0 \mathrm{a}$ \\
\hline$S$ & $20.0 \mathrm{~b}$ & $31.8 \mathrm{~b}$ & $31.5 \mathrm{~b}$ & $0 \mathrm{a}$ & $16.5 \mathrm{~d}$ & $20.3 \mathrm{a}$ \\
\hline \multicolumn{7}{|c|}{ Sample X } \\
\hline $\mathrm{TP}$ & $17.0 \mathrm{a}$ & $20.8 \mathrm{a}$ & $53.8 \mathrm{~d}$ & $0 \mathrm{a}$ & $0 \mathrm{a}$ & $25.5 \mathrm{~b}$ \\
\hline TP-D & $27.5 \mathrm{~b}$ & $32.0 \mathrm{~b}$ & $35.5 \mathrm{c}$ & $0.3 \mathrm{a}$ & $0 \mathrm{a}$ & $32.3 \mathrm{~b}$ \\
\hline $\mathrm{PP}$ & $54.8 \mathrm{~d}$ & $56.0 \mathrm{~d}$ & $26.5 \mathrm{~b}$ & $2.0 \mathrm{~b}$ & $2.3 \mathrm{~b}$ & $13.3 \mathrm{a}$ \\
\hline $\mathrm{RP}$ & $61.5 \mathrm{~d}$ & $63.3 \mathrm{~d}$ & $18.0 \mathrm{a}$ & $2.3 \mathrm{~b}$ & $2.3 \mathrm{~b}$ & $14.5 \mathrm{a}$ \\
\hline S & $35.8 \mathrm{c}$ & $43.3 c$ & $18.3 \mathrm{a}$ & $0.3 \mathrm{a}$ & $17.0 \mathrm{c}$ & $21.3 \mathrm{a}$ \\
\hline $\begin{array}{l}{ }^{*} \mathrm{TP}- \\
\mathrm{TP}-\mathrm{D}- \\
\mathrm{PP}-\mathrm{g} \\
\mathrm{RP}-\mathrm{g} \\
\mathrm{S}-\text { ger } \\
\text { Means }\end{array}$ & $\begin{array}{l}\text { op of blot } \\
\text { top of bl } \\
\text { en pleate } \\
\text { led blotte } \\
\text { e same let }\end{array}$ & es after se & ording & ncan' & & \\
\hline
\end{tabular}

highest percentages of abnormal infected seedlings and dead seeds, were observed in case of blotter tests in Petri dishes, both for disinfected and non-disinfected seeds (tab. 1). The only exception was sample VII, characterized by the worst quality among tested samples. For this sample, the lowest value of germination at the final count $(2.8 \%)$ was detected when seeds were grown in sand. In general, the values of germination at the first and final counts, determined by both tests in Petri dishes (TP-D and TP), did not differ significantly. Depending on the sample, the highest values of seed germination at the first and final count were observed after germination in sand (S) for sample I, in rolled paper (RP) for samples III and V, between pleated paper (PP) for samples II and IX, and after germination in rolled paper as well as between pleated paper for samples IV, VI, VII, VIII and X. However, in case of sample VII, values of seed germination at the final count, determined by means of RP and PP tests did not differ significantly from the values of this parameter found after germination in Petri dishes (TP-D and TP), and in case of samples IV and VIII, did not differ significantly from the values obtained after germination in sand. In nine out of ten samples examined, the highest percentage of abnormal diseased seedlings was detected in blotter tests in Petri dishes, both for disinfected and non-disinfected seeds. In other tests the number of these seedlings was significantly lower. Regardless of the method, all samples were characterized by a relatively low number of abnormal deformed seedlings. In general, after germination in sand the highest percentage of fresh seeds was recorded.

Many saprotrophic and potentially pathogenic fungi were identified on tested seeds (tab. 2). Among them: A. alternata, Cladosporium spp., Fusarium spp., Melanospora simplex (Corda) D. Hawksw, Mucor sp., Penicillium spp., Ulocladium consortiale (Thüm) E. Simmons and Verticillium spp. prevailed. Furthermore, A. alternata, Cladosporium spp., $\mathrm{Mu}$ cor sp., Penicillium spp., U. consortiale and Verticillium spp. were detected in all examined samples. Total seedling emergence ranged from 14.5 to $85.0 \%$, depending on sample. In seven of tested samples, the percentage of emerged seedlings was higher than the percentage of germination at the final count determined by the methods used (tab. 1 and 3). Nevertheless, during emergence test, disease symptoms appeared on $1.8-15.6 \%$ of seedlings (tab. 3). Mycological analysis showed that the emerged diseased seedlings were infested with several fungi, i.e.: Acremoniella atra (Corda), A. alternata, Alternaria sp., Aspergillus spp., A. flavus, A. niger, B. cinerea, Cladosporium spp., E. nigrum, Fusarium spp., M. simplex, Mucor spp., Penicillium spp., Phoma sp., Sarocladium sp., S. botrysoum, Thamnidium sp., Trichoderma spp., T. roseum, U. consortiale and Verticillium spp. (tab.4) Among them: A. alternata, Alternaria sp., Fusarium spp., Penicillium spp., Trichoderma spp., $U$. consortiale and Verticillium spp. were observed most frequently. Diseased seedlings of all samples, except sample VII, were abundantly infested with $A$. alternata. The percentage of diseased seedlings infested with this fungus ranged from 36.4 to 100.0. Apart from A. alternata, another fungus belonging to the genus Alternaria was also frequently identified. The percentage of seedlings infested with this fungus ranged from 20.0 to 85.7. The Alternaria sp. appeared on seedlings of most of the samples, 
Table 2

Occurrence of fungi on milk thistle seeds [\% of infested seeds]

\begin{tabular}{|c|c|c|c|c|c|c|c|c|c|c|}
\hline \multirow[b]{2}{*}{ Fungi } & \multicolumn{9}{|c|}{ Sample } & \multirow[b]{2}{*}{$\mathrm{X}$} \\
\hline & I & II & III & IV & $\mathrm{V}$ & VI & VII & VIII & IX & \\
\hline Acremoniella atra (Corda) Sacc. & 0 & 0 & 0 & 1.0 & 14.5 & 0.5 & 0 & 0 & 0 & 0 \\
\hline Alternaria alternata (Fr.) Keissl. & 70.5 & 87.0 & 20.0 & 64.0 & 77.0 & 62.0 & 20.0 & 89.0 & 18.0 & 70.5 \\
\hline Alternaria sp. & 0.5 & 1.0 & 17.0 & 3.0 & 11.5 & 1.0 & 0 & 0 & 0.5 & 0 \\
\hline Aspergillus spp. & 0.5 & 0.5 & 0 & 0 & 15.5 & 6.0 & 0 & 0 & 4.0 & 0 \\
\hline Aspergillus flavus Link & 0 & 0 & 0.5 & 0.5 & 0.5 & 1.0 & 0 & 0 & 0 & 1.0 \\
\hline Bipolaris spp. & 0 & 0 & 0 & 0 & 2.5 & 0 & 0 & 0 & 0 & 0.5 \\
\hline Botrytis cinerea Pers. & 0.5 & 0 & 0 & 1.0 & 0 & 0.5 & 0 & 1.5 & 0 & 0 \\
\hline Cladosporium spp. & 4.5 & 3.5 & 11.5 & 20.5 & 1.5 & 4.5 & 9.0 & 14.0 & 1.5 & 1.5 \\
\hline Epicoccum nigrum Link & 0.5 & 0 & 1.0 & 3.0 & 0 & 0 & 0 & 0 & 0 & 1.0 \\
\hline Fusarium spp. & 6.0 & 4.0 & 0.5 & 1.0 & 4.5 & 5.5 & 1.5 & 12.0 & 0 & 0 \\
\hline $\begin{array}{l}\text { Melanospora simplex (Corda) D. } \\
\text { Hawksw. }\end{array}$ & 42.5 & 25.5 & 0 & 21.5 & 33.0 & 3.5 & 0 & 0 & 49.0 & 0.5 \\
\hline Mucor spp. & 23.5 & 10.0 & 67.0 & 32.5 & 19.0 & 27.0 & 36.5 & 1.5 & 20.5 & 19.0 \\
\hline Penicillium spp. & 11.0 & 28.5 & 14.0 & 2.0 & 5.5 & 31.5 & 21.0 & 2.0 & 61.5 & 29.5 \\
\hline Phoma sp. & 0.5 & 0 & 0 & 0.5 & 0 & 0 & 0 & 0 & 0 & 0 \\
\hline Rhizopus stolonifer (Ehrenb.) Vuill. & 0 & 0 & 0.5 & 0 & 0.5 & 1.0 & 0 & 0 & 0 & 1.0 \\
\hline Sordaria sp. & 0 & 0 & 0 & 0 & 0 & 0 & 0 & 0 & 0.5 & 0.5 \\
\hline Stemphylium botryosum Wallr. & 0 & 0.5 & 0 & 0.5 & 1.5 & 0 & 0 & 1.0 & 0.5 & 0 \\
\hline Thamnidium sp. & 0 & 0 & 0 & 0 & 0 & 0 & 5.0 & 0 & 0 & 0.5 \\
\hline Trichoderma spp. & 0 & 0 & 1.0 & 0 & 0 & 0 & 0 & 0 & 0 & 0 \\
\hline Trichothecium roseum (Pers.) Link & 0 & 14.5 & 0 & 0 & 17.0 & 2.0 & 0 & 0 & 0 & 0 \\
\hline $\begin{array}{l}\text { Ulocladium consortiale (Thüm.) E.G. } \\
\text { Simmons }\end{array}$ & 48.5 & 38.0 & 34.0 & 66.5 & 27.5 & 26.0 & 6.0 & 63.5 & 18.0 & 65.5 \\
\hline Verticillium spp. & 33.5 & 8.0 & 2.0 & 22.5 & 27.5 & 2.5 & 2.0 & 26.5 & 2.0 & 6.0 \\
\hline
\end{tabular}

Table 3

Seedling emergence and the incidence of diseased seedlings

\begin{tabular}{lcc}
\hline Sample & Seedling emergence [\%] & $\begin{array}{c}\text { Diseased seedlings } \\
\text { [\% of emerged seedlings] }\end{array}$ \\
\hline I & 67.5 & 15.6 \\
\hline II & 73.5 & 12.3 \\
\hline III & 57.5 & 6.1 \\
\hline VV & 81.5 & 1.8 \\
\hline VI & 85.0 & 12.4 \\
\hline VII & 51.5 & 4.9 \\
\hline VIII & 14.5 & 3.5 \\
\hline IX & 77.0 & 10.4 \\
\hline X & 59.0 & 9.3 \\
\hline
\end{tabular}


Table 4

Occurrence of fungi on seedlings with disease symptoms in emergence test [\% of infected seedlings]

\begin{tabular}{|c|c|c|c|c|c|c|c|c|c|c|}
\hline \multirow[b]{2}{*}{ Fungi } & \multicolumn{8}{|c|}{ Sample } & \multirow[b]{2}{*}{ IX } & \multirow[b]{2}{*}{$\mathrm{X}$} \\
\hline & I & II & III & IV & V & VI & VII & VIII & & \\
\hline Acremoniella atra (Corda) Sacc. & 4.8 & 0 & 0 & 0 & 9.5 & 0 & 0 & 12.5 & 0 & 20.0 \\
\hline Alternaria alternata(Fr.) Keissl. & 81.0 & 72.2 & 85.7 & 100.0 & 85.7 & 60.0 & 0 & 81.3 & 36.4 & 80.0 \\
\hline Alternaria sp. & 62.0 & 66.7 & 85.7 & 33.3 & 71.4 & 20.0 & 0 & 25.0 & 45.5 & 0 \\
\hline Aspergillus spp. & 0 & 0 & 0 & 0 & 9.6 & 0 & 0 & 6.3 & 0 & 0 \\
\hline Aspergillus flavus Link & 0 & 0 & 0 & 0 & 0 & 0 & 0 & 0 & 9.1 & 0 \\
\hline Aspergillus niger Tiegh. & 0 & 5.6 & 0 & 0 & 0 & 0 & 0 & 0 & 0 & 0 \\
\hline Botrytis cinerea Pers. & 0 & 0 & 0 & 0 & 4.8 & 0 & 0 & 0 & 0 & 20.0 \\
\hline Cephalosporium sp. & 0 & 0 & 14.3 & 0 & 0 & 0 & 0 & 0 & 0 & 0 \\
\hline Cladosporium spp. & 4.8 & 5.6 & 0 & 33.3 & 9.5 & 0 & 0 & 18.8 & 0 & 20.0 \\
\hline Epicoccum nigrum Link & 0 & 5.6 & 0 & 0 & 0 & 0 & 0 & 0 & 0 & 0 \\
\hline Fusarium spp. & 81.5 & 66.7 & 57.1 & 33.3 & 28.6 & 20.0 & 100.0 & 25.0 & 0 & 20.0 \\
\hline Melanospora simplex (Corda) D. Hawksw. & 47.6 & 38.9 & 14.3 & 0 & 0 & 0 & 0 & 6.3 & 0 & 0 \\
\hline Mucor spp. & 19.0 & 11.1 & 14.3 & 0 & 4.8 & 20.0 & 0 & 0 & 9.1 & 0 \\
\hline Penicillium spp. & 4.8 & 11.1 & 42.9 & 66.7 & 23.8 & 20.0 & 0 & 18.8 & 36.4 & 60.0 \\
\hline Phoma sp. & 4.8 & 0 & 0 & 0 & 0 & 0 & 0 & 6.3 & 0 & 0 \\
\hline Stemphylium botryosum Wallr. & 0 & 5.6 & 0 & 0 & 0 & 0 & 0 & 0 & 0 & 0 \\
\hline Thamnidium sp. & 4.8 & 0 & 0 & 0 & 0 & 0 & 0 & 0 & 0 & 0 \\
\hline Trichoderma spp. & 28.6 & 22.2 & 0 & 0 & 23.8 & 80.0 & 100.0 & 12.5 & 36.4 & 80.0 \\
\hline Trichothecium roseum (Pers.) Link & 4.8 & 5.6 & 0 & 0 & 9.5 & 20.0 & 0 & 6.3 & 0 & 0 \\
\hline Ulocladium consortiale (Thüm.) E.G. Simmons & 23.8 & 50.0 & 71.4 & 33.3 & 52.4 & 20.0 & 0 & 75.0 & 63.6 & 80.0 \\
\hline Verticillium spp. & 4.8 & 0 & 14.3 & 0 & 52.4 & 60.0 & 0 & 68.8 & 36.6 & 80.0 \\
\hline
\end{tabular}

except sample VII and X. In turn, Botrytis cinerea was observed only on seedlings of samples $\mathrm{V}$ and $\mathrm{X}$ (4.8 and $20.0 \%$ of infected seedlings, respectively). Fungi of genus Fusarium were detected quite often on infected seedlings of all samples, except sample IX. Penicillium spp. and U. consortiale were not found only on seedlings of sample VII. In other samples the percentage of seedlings infested with these fungi ranged from 4.8 to 66.7 and from 20.0 to 80.0 , respectively. Fungi from genus Trichoderma were identified on $12.5-100.0 \%$ of seedlings, and only in samples III and IV these fungi were not detected. Verticillium spp. were observed on seedlings of most samples, however, the highest infestation with these fungi was recorded in sample X (80.0\%).

Highly significant positive correlations between seed germination at the final count evaluated in rolled blotter paper, between pleated blotter paper and in sand and seedling emergence were recorded (fig. 1). Moreover, a significant positive correlation between seed germination at the final count evaluated with blotter test in Petri dishes and seedling emergence was found.

\section{DISCUSSION}

Seed germination of ten milk thistle samples was compared using the standard methods described in the ISTA rules [4]. For most of the samples the highest values of germination at the final count were observed if seeds were germinated between pleated blotter paper, in rolled paper or in sand. On the other hand, the lowest values of this parameter, connected with the high percentages of diseased seedlings and dead seeds, were recorded after germination of milk thistle seeds on top of paper. Mycological analysis revealed that the seeds were infested with many saprotrophic and potentially pathogenic fungi. Presence of these fungi significantly influenced results of germination tests. Germination of seeds on top of blotter paper especially favoured the spread of fungal infection from diseased seedlings and dead seeds to healthy seedlings. In nine out of ten examined samples, the highest percentage of infected seedlings was detected in blotter tests in Petri dishes, both for disinfected and non-disinfected seeds. In other tests, the number of these seedlings was significantly lower. In case of poor milk thistle seed germination in blotter tests, associated with 


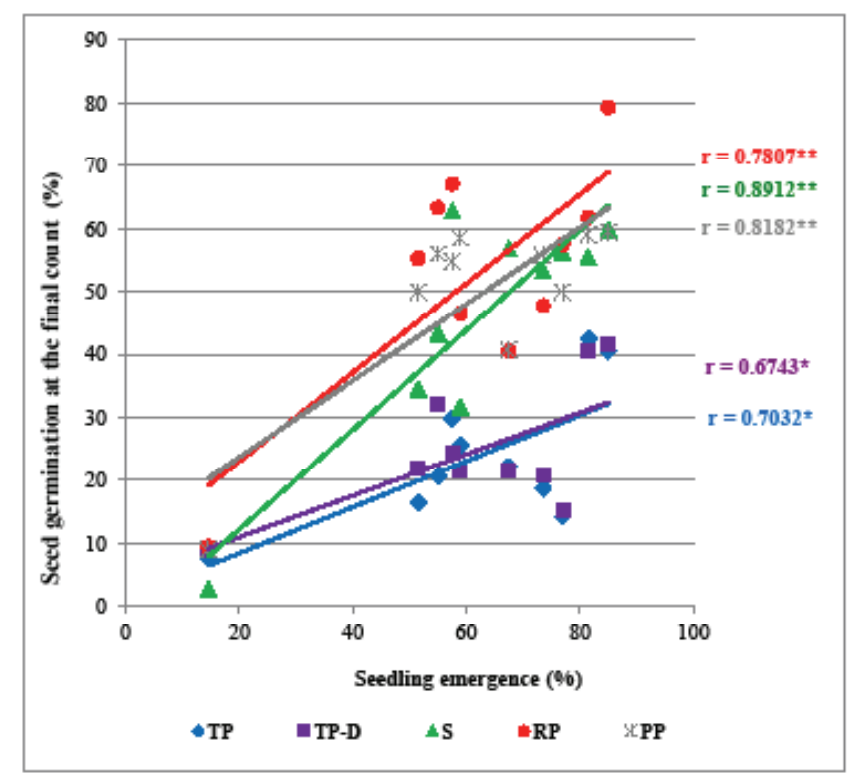

Figure 1

Relationships between seed germination at final count evaluated with different methods and seedling emergence

$r$ - Pearson correlation coefficient

* and ${ }^{* *}$ - significantly different at levels $\alpha=0.05$ and $\beta=0.01$

$\mathrm{TP}$ - germination on top of blotter paper in Petri dishes

TP-D - germination on top of blotter paper in Petri dishes after seed disinfection

$S$ - germination in sand

$\mathrm{RP}$ - germination in rolled blotter paper

PP - germination between pleated blotter paper

high percentage of diseased seedlings, ISTA recommends sand as an alternative medium for germination [4]. Mariappan et al. [12] reported that the percentage of normal seedlings in biofuel tree species was higher in sand than in blotter tests, and - in addition - on blotter media higher number of infected seedlings and dead seeds was recorded. According to Bahar [13], germination on top of blotter paper is effective in case of small seeds, while large ones should be germinated in sand or in rolled paper, and medium size seeds in rolled paper. The latter method is easier to handle than sand test and leads to easier and quicker development of seedlings. Loubser and van den Venter [14] observed that germination of tomato seeds in rolled blotter paper allows for easier evaluation of seedlings than germination on top of blotter or between folds of pleated blotter paper, and the cotyledons of seedlings in this test were visible at an early stage of incubation. The authors found that germination of seeds in Petri dishes, in limited space, could increase the number of seedlings with secondary loops. On the other hand the use of rolled paper inhibited looping of the hypocotyls and the occurrence of secondary fungal infections. In our experiment, germination in rolled blotter paper also favoured development of essentials organs of seedlings and limited spread of fungi, therefore allowed for faster and easier seedlings evaluation. Moreover, germination in rolled blotter, i.e. between paper sheets, requires much less space in germination facilities, which is important when large number of samples have to be tested in the laboratory at the same time [15].

For most of tested samples, total seedling emergence was higher than the values of germination at the final count determined by the methods used. The same phenomenon has been observed by $\mathrm{Du}$ cournau et al. [16, 17] who compared germination of sunflower and broad bean seeds on blotter, in sand and in organic media. The authors found that for both species normal germination in organic media was higher than on blotter or in sand. They explained these differences by a large number of abnormal fractured seedlings as a result of rapid water imbibition in sand and on blotter. In our experiment, a low number of abnormal deformated seedlings and a high percentage of infected seedlings observed in germination tests, lead us to a different conclusion. There is a possibility that after sowing, soil-borne microorganisms competed 
with seed-borne fungi, what resulted in a higher total seedling emergence. Nevertheless, some of these fungi were still able to affect seedlings health. Regardless of sample, seedlings with disease symptoms were abundantly infested with A. alternata. According to Cwalina-Ambroziak et al. [18], A. alternata is a potential pathogen of milk thistle. Moreover, this fungus is able to produce several mycotoxins, such as alternariol, alternariol monomethyl ether, altenuene, altertoxins I, II, III and tenuazonic acid $[19,20]$. Bottalico and Logrieco [21] reported that tenuazonic acid exhibited a severe growth inhibiting activity both on roots and on shoots of germinating tomato seeds. The authors observed that the phytotoxic effect was highly correlated to tenuazonic acid concentration. Alternaria alternata was frequently observed on milk thistle seeds by Rosińska et al. and Machowicz-Stefaniak and Zimowska [5, 6]. The presence of this fungus on seeds and infected emerged seedlings confirms its pathogenicity for the milk thistle plant. Another species from genus Alternaria was also identified on the diseased seedlings. The appearance of spores and conidiophores of this fungus corresponds to the description of Alternaria silybi (Gannibal), isolated from milk thistle leaves by Gannibal [22]. Alternaria sp. infested a relatively low number of seeds, but it was found frequently on infected seedlings in emergence test. This phenomenon may be a proof of its pathogenicity. According to Cwalina-Ambroziak [18], also B. cinerea and fungi of the genera Fusarium and Phoma are potential pathogens of milk thistle. Fusarium spp. were often observed on infected seedlings of all samples, but B. cinerea and Phoma sp. occurred only on seedlings of two samples.

The results of all applied germination tests (germination at the final count) were positively correlated with results of emergence tests, however, highly significant positive correlations were noted between germination at final count and seedlings emergence, if seeds were germinated in sand, between pleated blotter paper and in rolled blotter paper. Our experience, as well as other authors' observations [15], confirmed that among these tests, evaluation of seeds in rolled blotter paper is the most practical and easiest to perform.

\section{CONCLUSIONS}

1. A significant positive correlation between seed germination at the final count, evaluated with blotter test in Petri dishes, and seedling emergence was found, however, highly significant positive correlations were noted between germination at the final count evaluated in rolled blotter paper, between pleated blotter paper and in sand, and seedling emergence.

2. Germination of seeds in rolled blotter paper favoured the development of seedlings, limited spread of fungi and secondary infections, and the test was the easiest to perform.

3. Seeds and diseased seedlings were commonly infested with Alternaria spp. and Fusarium spp. - the fungi, which can be pathogenic to milk thistle plants.

Conflict of interest: Authors declare no conflict of interest.

\section{REFERENCES}

1. Morazzoni P, Bombardelli E. Silybum marianum (Carduus marianus). Fitoterapia 1995; 64:3-42.

2. Andrzejewska J, Martinelli T, Sadowska K. Silybum marianum: non-medical exploitation of the species. Ann Appl Biol 2015; 167:285-297. doi: http://dx.doi.org/ 10.1111/aab.12232

3. Seidler-Łożykowska K. Medicinal plant seeds as an element of increasing biodiversity of grassland. SALVARE - Regional workshop in Poland, Poznań University of Life Sciences 2009; 45-47.

4. ISTA. International Rules for Seed Testing. International Seed Testing Association, Bassersdorf, Switzerland 2012.

5. Rosińska A, Jarosz M, Szopińska D, Dorna $\mathrm{H}$, Tylkowska K. Comparison of methods for detecting fungi in Silybum marianum (L.) Gaertn. seeds. Folia Hort 2013; 25(2):107-115. doi: http://dx.doi.org/10.2478/fhort-2013-0012

6. Machowicz-Stefaniak Z, Zimowska B. Grzyby przenoszone przez materiał siewny roślin zielarskich [Fungi transmitted with seeds of herbal plants]. Acta Agrobot 2000; 52(2):25-38.

7. Malone JP, Muskett AE. Seed borne-fungi. Description of 77 fungus species. The Interna- 
tional Seed Testing Association, Zurych, Switzerland 1964.

8. Watanabe T. Pictorial atlas of soil and seed fungi morphologies of cultured fungi and key to species. CRC PRESS, Boca Raton, London, New York, Washington 2002.

9. Mathur SB, Kongsdal O. Common laboratory seed health testing methods for detecting fungi. The International Seed Testing Association, Basserdorf, Switzerland 2003.

10. Kala R. Statystyka dla przyrodników. Wyd. AR, Poznań 2002.

11. Jalink H, van der Schoor R. Seed Calculator 2.1. License number: 100200122. 1999; Plant Research International. Wageningen, The Netherlands.

12. Mariappan N, Srimathi P, Sundaramoorthi L, Sudhakar K. Effect of growing media on seed germination and vigor in biofuel tree species. J For Res 2014; 25(4):909-913. doi: http://dx.doi. org/ 10.1007/s11676-014-0484-8

13. Bahar N. Effect of media on seed germination of Cupaniopsis anacardioides (A. Rich.) Radlk Ind J For 2008; 31(1):137-139.

14. Loubser WA, van den Venter HA. The optimal germination substratum for tomato (Lycopersicon lycopersicum (L.) Karst. ex Farw.) seed testing. S Afr J Plant Soil 1990; 7(3):201-204. doi: http://dx.doi.org/10.1080/02571862.1990.1063 4568

15. Ripka Z. New method for germination of Brassica spp. and Sinapsis alba seeds. Seed Testing International 2009; 137:35-37.
16. Ducournau S, Garreau P, Léchappé J. Effect of temperature and growing media on sunflower germination. Seed Test Int 2008; 135:34-36.

17. Ducournau S, Garreau P, Léchappé J. Use of organic growing media as a primary substrate for germination of Vicia faba seeds. Seed Test Int 2009; 137:37-39.

18. Cwalina-Ambroziak B, Wierzbowska J, Damszel M, Bowszys T. The effect of mineral fertilization on achenes yield and fungal communities isolated from the stems of milk thistle Silybum marianum (L.) Gaertner. Acta Sci Pol Hortorum Cultus 2012; 11(4):157-168.

19. Dorna H. Wybrane gatunki grzybów rodzaju Alternaria w nasionach marchwi - ich lokalizacja, toksynotwórczość, wpływ na wigor i kiełkowanie nasion oraz wschody [Selected species of genus Alternaria on carrot seeds - their location, toxigenicity, effect on seed vigour and germination and seedling emergence]. Wyd. AR-Poznań 2007.

20. Logrieco A, Morettic A, Solfrizzo M. Alternaria toxins and plant diseases: an overview of origin, occurrence and risks. World Mycotoxin J 2009; 2(2):129-140. doi: http://dx.doi. org/10.3920/WMJ2009.1145

21. Bottalico A, Logrieco A. Alternaria plant diseases in Mediterranean countries and associated mycotoxins. In: Alternaria: biology, plant diseases and metabolites. J. Chełkowski, A. Visconti (eds.). Amsterdam 1992; 209-232.

22. Gannibal PB. Taxonomic studies of Alternaria from Russia: new species on Asteraceae. Mycotaxon 2010; 114:109-114. 